\title{
HER2 amplification detected in the circulating DNA of patients with gastric cancer: a retrospective pilot study
}

\author{
Katsutoshi Shoda $\cdot$ Kiyoshi Masuda $\cdot$ Daisuke Ichikawa $\cdot$ \\ Tomohiro Arita • Yuko Miyakami • Miki Watanabe • \\ Hirotaka Konishi · Issei Imoto • Eigo Otsuji
}

Received: 26 April 2014/ Accepted: 14 September 2014/Published online: 17 October 2014

(c) The International Gastric Cancer Association and The Japanese Gastric Cancer Association 2014

\begin{abstract}
Background We used real-time quantitative polymerase chain reaction (rqPCR) to detect human epidermal growth factor receptor 2 (HER2) amplification in the circulating cell-free DNA (cfDNA) of patients with gastric cancer (GC), which shows the spatial and temporal intrinsic heterogeneity of HER2 expression/copy number during progression, for liquid biopsy and treatment monitoring.

Methods We first enrolled 52 patients with advanced GC who underwent surgery and 40 healthy volunteers. For patients with GC, plasma cfDNA was obtained before surgery (43 patients) and during postoperative treatment (nine of 43 patients). After ribonuclease P RNA component $\mathrm{H} 1$ (RPPH1) had been selected as a reference gene for $H E R 2 \mathrm{CN}$ assessment by rqPCR in GC tumours and plasma, plasma HER2-to-RPPH1 ratios were determined retrospectively in a development cohort and an additional independent validation cohort.
\end{abstract}

Electronic supplementary material The online version of this article (doi:10.1007/s10120-014-0432-5) contains supplementary material, which is available to authorized users.

K. Shoda $\cdot$ D. Ichikawa $(\varangle) \cdot$ T. Arita $\cdot$ H. Konishi $\cdot$ E. Otsuji Division of Digestive Surgery, Department of Surgery, Kyoto Prefectural University of Medicine, 465 Kawaramach Hirokoji Kajiicho, Kamigyo-ku, Kyoto 602-8566, Japan

e-mail: ichikawa@koto.kpu-m.ac.jp

K. Shoda $\cdot$ K. Masuda $\cdot$ I. Imoto $(\bowtie)$

Department of Human Genetics, Institute of Health Biosciences, University of Tokushima Graduate School,

3-18-15 Kuramoto-cho, Tokushima 770-8503, Japan

e-mail: issehgen@tokushima-u.ac.jp

Y. Miyakami · M. Watanabe

Student Laboratory, Faculty of Medicine, University

of Tokushima, Tokushima, Japan
Results The HER2-to-RPPH1 ratio of GC tissues determined by rqPCR was concordant with routinely determined HER2 status. The plasma HER2-to-RPPH1 ratio was significantly higher for patients with HER2-positive tumours than for those with HER2-negative tumours. The sensitivity and specificity of the plasma $H E R 2$-to- $R P P H 1$ ratio test were 0.539 and 0.967 , respectively, in the development cohort, and 0.667 and 1.000 , respectively, in the validation cohort. HER2 amplifications acquired and lost during tumour progression and treatment, respectively, were apparently detected by repeated assessments of plasma HER2-to-RPPHI ratios during postoperative treatment.

Conclusion Our preliminary data demonstrated the potential clinical use of circulating cfDNA to detect HER2 amplification as a therapeutic marker to detect and monitor HER $2 \mathrm{CN}$ status for effective molecular targeted therapy in patients with GC.

Keywords HER2 gene amplification - Gastric cancer . Cell-free DNA · Trastuzumab · Liquid biopsy

\section{Introduction}

Gastric cancer (GC) is one of the most commonly diagnosed cancers and is the sixth-leading cause of cancerrelated deaths worldwide [1]. Although surgery remains the mainstay treatment for GC, GC patients often present with advanced, inoperable disease for which conventional chemotherapy reagents have limited success [2]. Thus, there is a need for more effective treatment options.

Human epidermal growth factor receptor 2 (HER2) is a transmembrane receptor tyrosine kinase. Its oncogenic activation (e.g. amplification) results in its protein overexpression and subsequent abnormal cell signalling, which 
contributes to cancer progression. HER2 overexpression/ amplification was reported in 7-34 \% of patients with GC, and in a meta-analysis it was associated with more aggressive disease and poorer survival [3, 4]. A recent phase III trial of trastuzumab for patients with HER2-positive advanced GC (ToGA trial) showed that the addition of trastuzumab to chemotherapy produced a statistically significant benefit in terms of overall survival versus chemotherapy alone [3]. On the basis of these results, the European Medicines Agency approved trastuzumab for the treatment of metastatic adenocarcinomas of the stomach in 2010. However, it noted that trastuzumab should be used only for patients with metastatic GC whose tumours overexpress HER2 as routinely defined by an immunohistochemistry (IHC) score of $3+$ or $2+$ with a positive fluorescent in situ hybridization (FISH) result. Therefore, an accurate diagnosis of HER2 status in GC has become increasingly important for deciding on the treatment in the clinical setting.

Two important issues for HER2-targeted therapy in GC are (1) inaccurate assessments of HER2 status owing to intratumoral heterogeneity [5-7] or genetic differentiation accompanied by neoplastic progression, which results in inappropriately choosing patients for HER2-targeted therapy, and (2) therapeutic resistance owing to selection of HER2-amplified subclones accompanied by HER2-targeted therapy. Indeed, intratumoral HER2 heterogeneity is more frequent in GC than in breast cancer (BC) [5, 8], and the overall rate of response to HER2-targeted therapy in patients with HER2-positive GC is $47.3 \%$ [3], indicating that there are a considerable proportion of these patients who may be refractory to HER2 inhibition, even when the drug target is present.

Analysis of plasma or serum circulating cell-free DNA (cfDNA), one type of 'liquid biopsy', has attracted clinicians' interest as a new approach that is suitable for repeated measurements of HER2 amplification for the purpose of treatment monitoring and without the need for an invasive biopsy [9-12]. In a study of HER2-positive metastatic $\mathrm{BC}$, reduced $H E R 2$ amplification detected in plasma after first-line trastuzumab therapy in combination with chemotherapy was correlated with improved response and overall survival [13]. Although detection of amplification or methylation of cancer-related genes using circulating cfDNA in GC has also been reported [14, 15], no studies have examined the dynamics of plasma HER2 amplification in cfDNA before and after surgery and during adjuvant chemotherapy with or without trastuzumab in GC [7].

The dynamics of HER2 amplification detected in circulating cfDNA as determined by real-time quantitative polymerase chain reaction (rqPCR) may be of diagnostic and prognostic significance. In this pilot study, we focused on a type of 'liquid biopsy' using repeated plasma cfDNA sampling, and assessed the performance of rqPCR-based HER2 copy number (CNs) analysis as a non-invasive approach in advanced GC for the purpose of better molecular prediction of therapeutic effects and treatment monitoring.

\section{Materials and methods}

Patients and samples

Our study protocol was approved by the local ethics committee and was conducted in accordance with the Declaration of Helsinki. All subjects gave their written informed consent prior to their participation. Samples were blinded for analysis and patients understood that the results would not be made available to them. The development cohort (Table 1) and the independent validation cohort to assess the cut-off value for plasma rqPCR-based $H E R 2 \mathrm{CN}$ analysis (Table S1) included 52 and 25 patients with GC, respectively, who underwent surgery and had IHC HER2 score determinations between January 2008 and July 2013 at the Kyoto Prefectural University of Medicine Hospital (Fig. 1). Among these patients, preoperative plasma samples were collected from 43 patients (Table 2). For 48 patients, resected cancer specimens were obtained and fixed in buffered formalin and embedded in paraffin for routine pathology examinations (Table S2). Both preoperative plasma samples and tumour specimens were obtained from 39 patients. Control plasma samples were also obtained from 40 adult healthy volunteers by standard antecubital venous puncture.

Patient demographic data and details for tumour recurrence and subsequent management were recorded. Computed tomography (CT) was performed and the findings were reviewed in a blinded manner to document treatment responses according to the Response Evaluation Criteria in Solid Tumors (RECIST), version 1.1 [16]. Pathological classification of tumours was determined according to Union for International Cancer Control classifications [17]. Tissue HER2 status was determined with formalin-fixed and paraffin-embedded (FFPE) samples using routine IHC and FISH methods. Following the American Society of Clinical Oncologists/College of American Pathologists guideline recommendations, an IHC scores of both 0 and $1+$ were considered HER2 negative, whereas an IHC score of $3+$ was defined as HER2 positive. An IHC score of $2+$ was considered equivocal; a tumour was considered to be HER 2 positive if it had an IHC score of $2+$ plus FISH with a threshold ratio of more than 2.0 between the $H E R 2 \mathrm{CN}$ and chromosome 17 centromere (CEP17). Trastuzumab therapy was routinely administered to HER2-positive patients; however, two HER2-positive patients with GC refused this therapy and one HER2-negative patient with GC received this therapy (Table 1 ). 
Table 1 Clinicopathological features and their relationship with the tumour muman epidermal growth factor receptor 2 (HER2) status in 52 patients with gas cancer from the development cohort
${ }^{a}$ Tumour HER2 status was determined by an immunohistochemistry (IHC) score of 3 or an IHC score of 2 with a positive fluorescent in situ hybridization result as described in the text.

b $P$ values are from the $\chi^{2}$ test or Fisher's exact test, and were statically significant at less than 0.05 . Statistically significant values are given in italics.

${ }^{c}$ Mean \pm standard deviation

d 'Differentiated' corresponds to tubular adenocarcinoma or papillary adenocarcinoma, and 'undifferentiated' corresponds to poorly differentiated adenocarcinoma, signet-ring carcinoma or mucinous adenocarcinoma.

e Disease stage was defined in accordance with the Union for International Cancer Control seventh tumour-lymph nodemetastasis (TNM) classification using surgical-pathologic findings.

${ }^{\mathrm{f}}$ Trastuzumab therapy for patients with HER2-positive gastric cancer was performed on the basis of the European Medicines Agency criteria.

\begin{tabular}{|c|c|c|c|c|}
\hline \multirow[t]{2}{*}{ Variables } & \multirow[t]{2}{*}{ Number } & \multicolumn{3}{|c|}{ Tumour HER2 status ${ }^{\mathrm{a}}$} \\
\hline & & Negative $(\%)$ & Positive $(\%)$ & $P^{\mathrm{b}}$ \\
\hline Total & 52 & $38(73.1)$ & $14(26.9)$ & \\
\hline \multicolumn{5}{|l|}{ Gender } \\
\hline Male & 31 & $23(74.2)$ & $8(25.8)$ & \multirow[t]{2}{*}{0.8258} \\
\hline Female & 21 & $15(71.4)$ & $6(28.6)$ & \\
\hline Age $(\text { years })^{c}$ & & $65.6 \pm 12.1$ & $64.9 \pm 8.7$ & 0.7027 \\
\hline \multicolumn{5}{|c|}{ Histopathologically predominant $\mathrm{d}^{\mathrm{d}}$} \\
\hline Differentiated & 19 & $12(63.2)$ & $7(36.8)$ & \multirow[t]{2}{*}{0.2261} \\
\hline Undifferentiated & 33 & $26(78.8)$ & $7(21.2)$ & \\
\hline $\operatorname{Size}^{\mathrm{c}}$ & & $96.8 \pm 53.1$ & $92.4 \pm 41.9$ & 0.8527 \\
\hline \multicolumn{5}{|l|}{ T stage $^{\mathrm{e}}$} \\
\hline $\mathrm{T} 2 / 3$ & 17 & $12(70.6)$ & $5(29.4)$ & \multirow[t]{2}{*}{0.7790} \\
\hline $\mathrm{T} 4$ & 35 & $26(74.3)$ & $9(25.7)$ & \\
\hline \multicolumn{5}{|l|}{$\mathrm{N}$ stage $^{\mathrm{e}}$} \\
\hline No & 11 & $10(90.9)$ & $1(9.1)$ & \multirow[t]{3}{*}{0.0001} \\
\hline $\mathrm{N} 1 / 2$ & 27 & $24(88.9)$ & $3(11.1)$ & \\
\hline N3 & 14 & 4 (28.6) & $10(71.4)$ & \\
\hline \multicolumn{5}{|l|}{ Stage $^{\mathrm{e}}$} \\
\hline II & 10 & $9(90.0)$ & $1(10.0)$ & \multirow[t]{3}{*}{0.3168} \\
\hline III & 40 & $28(70.0)$ & $12(30.0)$ & \\
\hline IV & 2 & $1(50.0)$ & $1(50.0)$ & \\
\hline \multicolumn{5}{|c|}{ Lymphatic invasion } \\
\hline $0-2$ & 12 & $10(83.3)$ & $2(16.7)$ & \multirow[t]{2}{*}{0.3437} \\
\hline 3 & 40 & $28(70.0)$ & $12(30.0)$ & \\
\hline \multicolumn{5}{|l|}{ Venous invasion } \\
\hline $0-2$ & 40 & $29(72.5)$ & $11(27.5)$ & \multirow[t]{2}{*}{0.8633} \\
\hline 3 & 12 & $9(75.0)$ & $3(25.0)$ & \\
\hline \multicolumn{5}{|c|}{ HER2 expression by IHC } \\
\hline 0 & 16 & $16(100.0)$ & $0(0.0)$ & \multirow[t]{4}{*}{$<0.0001$} \\
\hline 1 & 15 & $15(100.0)$ & $0(0.0)$ & \\
\hline 2 & 9 & $7(77.8)$ & $2(22.2)$ & \\
\hline 3 & 12 & $0(0.0)$ & $12(100.0)$ & \\
\hline \multicolumn{5}{|c|}{ Neoadjuvant chemotherapy } \\
\hline Present & 5 & $2(40.0)$ & $3(60.0)$ & \multirow[t]{2}{*}{0.1002} \\
\hline Absent & 47 & $36(76.6)$ & $11(23.4)$ & \\
\hline \multicolumn{5}{|c|}{ Trastuzumab therapy ${ }^{\mathrm{f}}$} \\
\hline Present & 13 & $1(7.7)$ & $12(92.3)$ & \multirow[t]{2}{*}{$<0.0001$} \\
\hline Absent & 39 & $37(94.9)$ & $2(5.1)$ & \\
\hline
\end{tabular}

To assess rqPCR-based methods, genomic DNA from GC cell lines (Table S3), SK-BR-3 BC cells (American Type Culture Collection, Manassas, VA, USA) and normal leucocytes (Promega, Madison, WI, USA) were used.

Preparation and DNA isolation from plasma and tissues samples

A 3-ml EDTA-blood sample was obtained from each patient before surgery and from 40 healthy volunteers.
In nine of 43 patients from the development cohort and 11 of 25 patients from the validation cohort, samples were also collected after surgery and/or during adjuvant therapy. Plasma was immediately separated from the cellular fraction using a three-spin protocol as described elsewhere [18], and was stored at $-80{ }^{\circ} \mathrm{C}$ until further processing. Cell-free DNA (cfDNA) was isolated from $200 \mu \mathrm{l}$ of a plasma sample with a QIAamp MinElute virus spin kit (Qiagen, Hilden, Germany). 
Fig. 1 Study design. $c f D N A$ cell-free DNA, $C N$ copy number, $F F P E$ formalin fixed and paraffin embedded, $G C$ gastric cancer, HER2 human epidermal growth factor receptor 2, rqPCR real-time quantitative polymerase chain reaction

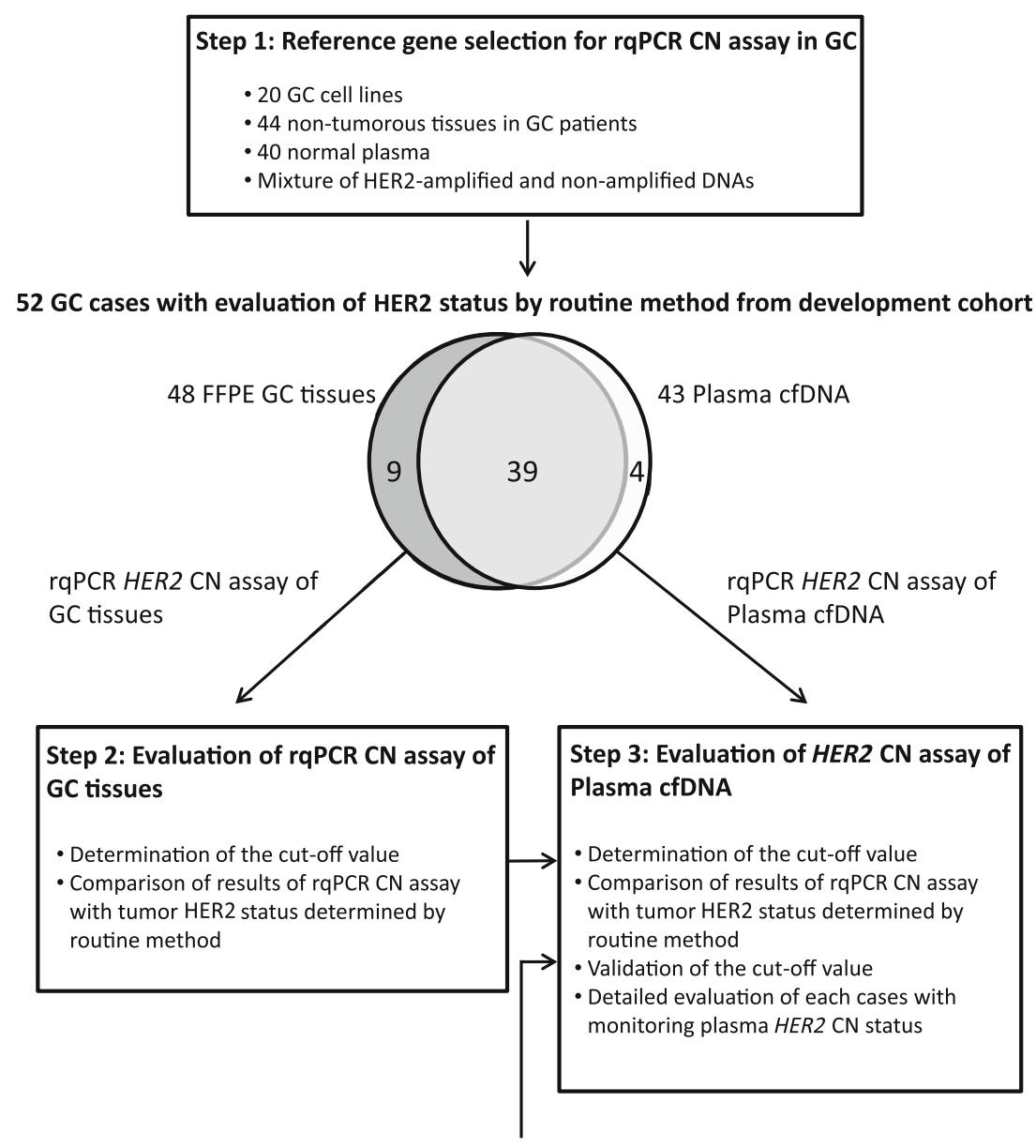

$25 \mathrm{GC}$ cases with evaluation of HER2 status by routine method from validation cohort
Genomic DNA of cancerous and adjacent non-cancerous gastric tissues was extracted from three slices $(5-\mu \mathrm{m}$ thick) of FFPE tissues using a ReliaPrep FFPE gDNA Miniprep system (Promega).

Quantitative analysis of HER2 CNs using rqPCR

All rqPCR amplifications were performed in triplicate using an Applied Biosystems 7500 real-time PCR system (Life Technologies, Carlsbad, CA, USA). The final PCR mixture $(10 \mu \mathrm{l})$ contained $5 \mu \mathrm{l}$ of TaqMan universal PCR master mix (Life Technologies), $0.5 \mu 1$ of TaqMan CN assay (Life Technologies) for HER2 (Hs05499477_cn), ribonuclease P RNA component $\mathrm{H} 1$ (RPPHI) (TaqMan reference assay), TAOK1 (Hs02294672_cn) or EFTUD2 (Hs00364067_cn) and $4.5 \mu \mathrm{l}$ of a DNA sample.

To determine HER2 gene amplification (HER2 to reference gene ratio), the $\Delta C_{\mathrm{t}}$ value was determined (average $C_{\mathrm{t}}$ value of the target gene minus the average $C_{\mathrm{t}}$ value of the reference gene) and was used to calculate the $\Delta \Delta C_{\mathrm{t}}$ value for each DNA sample with a mean relative quantitation (RQ) value. The RQ values for $\mathrm{GC}$ tissues and plasma cfDNA from patients with GC were derived from 44 non-tumour gastric tissues $[R Q=1.00 \pm 0.2953$; mean \pm standard deviation (SD)] and a panel of plasma cfDNA samples from 40 healthy volunteers $(R Q=1.00 \pm 0.3162)$, respectively, used as experimental calibrators. RQ values were calculated from $2^{-\Delta \Delta C \mathrm{t}}$. A $Z$ score for plasma sample analysis was calculated as follows: $Z$ score $=(\mathrm{RQ}$ patient plasma sample $-\mathrm{RQ}$ average normal plasma panel)/average SD of normal plasma panel.

Identifying a reference region

We obtained $\mathrm{CN}$ data as determined by microarray (Affymetrix SNP6.0 array) from the publically available Cancer Cell Line Encyclopedia (CCLE) database (BROAD Institute; http://www.broadinstitute.org/) for $20 \mathrm{GC}$ cell lines, which were available for our rqPCR analysis. To assess candidate reference genes for rqPCR-based HER2 $\mathrm{CN}$ testing, we compared our rqPCR results with $\mathrm{CN}$ data from the CCLE database in cell lines, and also performed rqPCR for normal gastric tissues and normal control plasma cfDNA. 
Table 2 Clinicopathological features of 43 gastric cancer patients with analysis of plasma real-time quantitative polymerase chain reaction (rqPCR) HER2-to-RPPH1 copy number ratio from the development cohort

\begin{tabular}{|c|c|c|c|c|c|c|c|c|c|c|c|}
\hline \multirow[t]{3}{*}{ Variables } & \multirow[t]{3}{*}{ Number } & \multicolumn{5}{|c|}{ Tumour HER2 status ${ }^{\mathrm{a}}$} & \multicolumn{5}{|c|}{ Plasma $H E R 2$ amplication ${ }^{\mathrm{b}}$} \\
\hline & & \multicolumn{2}{|c|}{ Negative } & \multicolumn{2}{|c|}{ Positive } & \multirow[t]{2}{*}{$P^{\mathrm{c}}$} & \multicolumn{2}{|c|}{ Absent } & \multicolumn{2}{|c|}{ Present } & \multirow[t]{2}{*}{$P^{\mathrm{c}}$} \\
\hline & & $n$ & $\%$ & $n$ & $\%$ & & $n$ & $\%$ & $n$ & $\%$ & \\
\hline Total & 43 & 30 & 69.8 & 13 & 30.2 & & 35 & 81.4 & 8 & 18.6 & \\
\hline \multicolumn{12}{|l|}{ Gender } \\
\hline Male & 25 & 17 & 68.0 & 8 & 32.0 & \multirow[t]{2}{*}{0.7656} & 22 & 88.0 & 3 & 12.0 & \multirow[t]{2}{*}{0.1921} \\
\hline Female & 18 & 13 & 72.2 & 5 & 27.8 & & 13 & 72.2 & 5 & 27.8 & \\
\hline Age (years) ${ }^{\mathrm{d}}$ & & \multicolumn{2}{|c|}{$66.4 \pm 10.7$} & \multicolumn{2}{|c|}{$65.9 \pm 8.1$} & 0.8635 & \multicolumn{2}{|c|}{$66.5 \pm 10.3$} & \multicolumn{2}{|c|}{$65.4 \pm 8.3$} & 0.7669 \\
\hline \multicolumn{12}{|c|}{ Histopathologically predominant $\mathrm{e}^{\mathrm{e}}$} \\
\hline Differentiated & 16 & 10 & 62.5 & 6 & 37.5 & \multirow[t]{2}{*}{0.4275} & 12 & 75.0 & 4 & 25.0 & \multirow[t]{2}{*}{0.4127} \\
\hline Undifferentiated & 27 & 20 & 74.1 & 7 & 25.9 & & 23 & 85.2 & 4 & 14.8 & \\
\hline Size $^{\mathrm{d}}$ & & \multicolumn{2}{|c|}{$95.0 \pm 51.5$} & \multicolumn{2}{|c|}{$98.0 \pm 41.3$} & 0.5620 & \multicolumn{2}{|c|}{$89.5 \pm 49.2$} & \multicolumn{2}{|c|}{$130.0 \pm 22.4$} & 0.0236 \\
\hline T stage $\mathrm{f}^{\mathrm{f}}$ & & & & & & & & & & & \\
\hline $\mathrm{T} 2 / 3$ & 13 & 9 & 69.2 & 4 & 30.8 & 0.9598 & 11 & 84.6 & 2 & 15.4 & 0.7172 \\
\hline $\mathrm{T} 4$ & 30 & 21 & 70.0 & 9 & 30.0 & & 24 & 80.0 & 6 & 20.0 & \\
\hline No & 7 & 6 & 85.7 & 1 & 14.3 & 0.0005 & 6 & 85.7 & 1 & 14.3 & 0.0637 \\
\hline $\mathrm{N} 1 / 2$ & 24 & 21 & 87.5 & 3 & 12.5 & & 22 & 91.7 & 2 & 8.3 & \\
\hline N3 & 12 & 3 & 25.0 & 9 & 75.0 & & 7 & 58.3 & 5 & 41.7 & \\
\hline Stage $^{\mathrm{f}}$ & & & & & & & & & & & \\
\hline II & 6 & 5 & 83.3 & 1 & 16.6 & 0.6221 & 6 & 100.0 & 0 & 0.0 & 0.1723 \\
\hline III & 35 & 24 & 68.6 & 11 & 31.4 & & 28 & 80.0 & 7 & 20.0 & \\
\hline IV & 2 & 1 & 50.0 & 1 & 50.0 & & 1 & 50.0 & 1 & 50.0 & \\
\hline $0-2$ & 10 & 9 & 90.0 & 1 & 10.0 & 0.0865 & 10 & 100.0 & 0 & 0.0 & 0.0291 \\
\hline 3 & 33 & 21 & 63.6 & 12 & 36.4 & & 25 & 75.8 & 8 & 24.2 & \\
\hline Venous invasion & & & & & & & & & & & \\
\hline $0-2$ & 33 & 23 & 69.7 & 10 & 30.3 & 0.9854 & 26 & 78.8 & 7 & 21.2 & 0.3993 \\
\hline 3 & 10 & 7 & 70.0 & 3 & 30.0 & & 9 & 90.0 & 1 & 10.0 & \\
\hline HER2 expression $\mathrm{t}$ & & & & & & & & & & & \\
\hline 0 & 11 & 11 & 100.0 & 0 & 0.0 & $<0.0001$ & 11 & 100.0 & 0 & 0.0 & 0.0007 \\
\hline 1 & 13 & 13 & 100.0 & 0 & 0.0 & & 13 & 100.0 & 0 & 0.0 & \\
\hline 2 & 8 & 6 & 75.0 & 2 & 25.0 & & 6 & 75.0 & 2 & 25.0 & \\
\hline 3 & 11 & 0 & 0.0 & 11 & 100.0 & & 5 & 45.5 & 6 & 55.5 & \\
\hline Neoadjuvant chem & rapy & & & & & & & & & & \\
\hline Present & 5 & 2 & 40.0 & 3 & 60.0 & 0.1406 & 3 & 60.0 & 2 & 40.0 & 0.2302 \\
\hline Absent & 38 & 28 & 73.7 & 10 & 26.3 & & 32 & 84.2 & 6 & 15.8 & \\
\hline Trastuzumab thera & & & & & & & & & & & \\
\hline Present & 13 & 1 & 7.7 & 12 & 92.3 & $<0.0001$ & 6 & 46.2 & 7 & 53.8 & 0.0001 \\
\hline Absent & 30 & 29 & 96.7 & 1 & 3.3 & & 29 & 96.7 & 1 & 3.3 & \\
\hline
\end{tabular}

a Tumour human epidermal growth factor receptor 2 (HER2) status was determined by an immunohistochemistry (IHC) score of 3 or an IHC score of 2 with a positive fluorescent in situ hybridization result as described in the text.

${ }^{\mathrm{b}}$ Plasma HER2 amplification was determined by the plasma rqPCR HER2-to-RPPH1 copy number ratio test with 3.05 as a cut-off value for the $Z$ score as described in "Quantitative analysis of HER2 CNs using rqPCR".

c $P$ values are from the $\chi^{2}$ or Fisher's exact test, and were statically significant at less than 0.05 . Statistically significant values are given in italics.

${ }^{\mathrm{d}}$ Mean \pm standard deviation

e 'Differentiated' corresponds to tubular adenocarcinoma or papillary adenocarcinoma, and 'undifferentiated' corresponds to poorly differentiated adenocarcinoma, signet-ring carcinoma or mucinous adenocarcinoma.

${ }^{\mathrm{f}}$ Disease stage was defined in accordance with the Union for International Cancer Control seventh tumour-lymph node-metastases (TNM) classification using surgical-pathologic findings.

g Trastuzumab therapy for patients with HER2-positive gastric cancer was performed on the basis of the European Medicines Agency criteria. 
a

Tissues
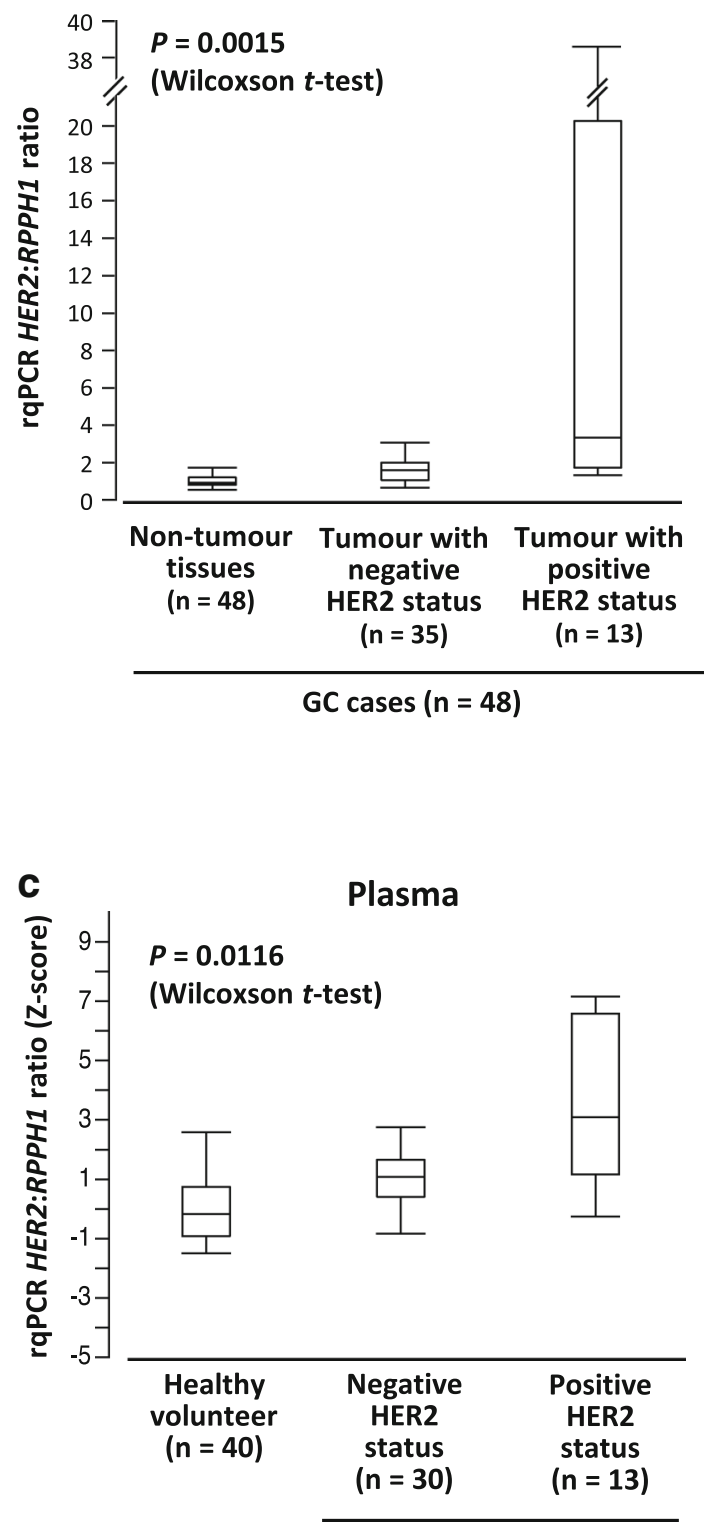

GC cases $(n=43)$

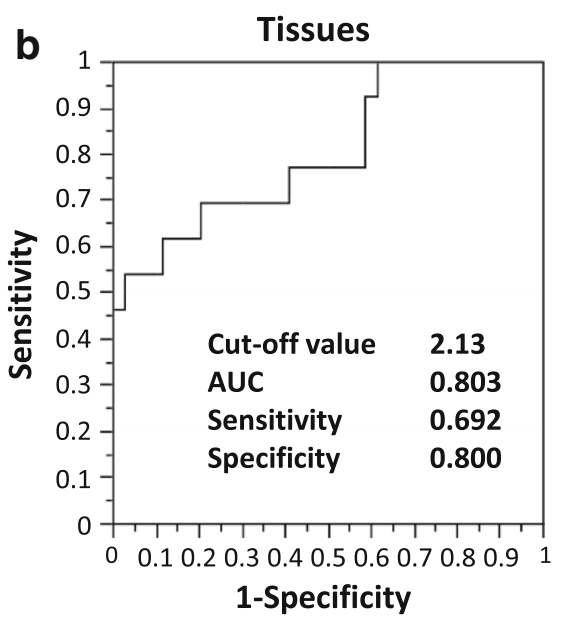

\begin{tabular}{c|c|cc}
\multicolumn{2}{l|}{} & \multicolumn{2}{c}{ Tumour HER2 status } \\
\cline { 3 - 4 } \multicolumn{2}{l|}{} & Positive & Negative \\
\hline $\begin{array}{c}\text { rqPCR HER2:RPPH1 } \\
\text { (Tissue, RQ) }\end{array}$ & $\geq 2.13$ & 9 & 7 \\
& $<2.13$ & 4 & 28
\end{tabular}

d Plasma

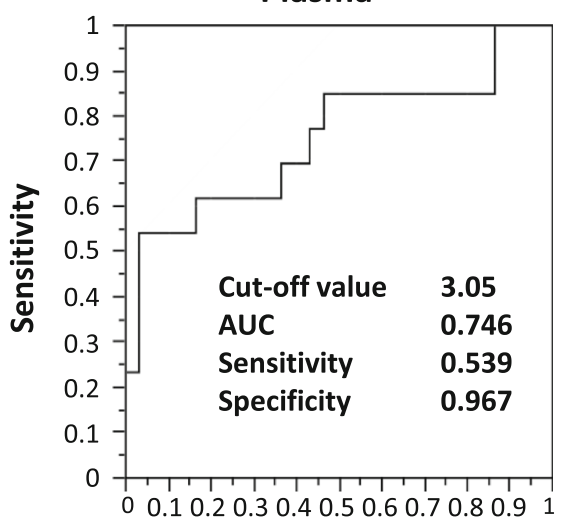

1-Specificity
Statistical analysis

Spearman correlation coefficients were determined to assess the associations between $\mathrm{CN}$ data determined by different methods. Non-parametric tests were used for subgroup comparisons (Wilcoxon $t$ test or Kruskal-Wallis test) and for comparisons between paired samples in each subgroup (Wilcoxon signed-rank test). The trends for tissue and plasma HER2-to-RPPH1 ratios across IHC HER2 scores were assessed using the Jonckheere-Terpstra test. Receiver operating characteristic (ROC) curves and the areas under the ROC curves (AUC) were used to assess the most appropriate cut-off values for detecting HER2-positive GC, which was determined using a Youden index [19]. A $\chi^{2}$ test or Fisher's exact test was used to assess associations between plasma HER2-to-RPPH1 ratio results and 
4Fig. 2 Correlations between tumour HER2 status and the rqPCR HER2-to-RPPH1 CN assay results of tissues and plasma samples in patients with GC. a Comparisons of tissue rqPCR HER2-to-RPPH1 $\mathrm{CN}$ ratios (relative quantitation, RQ) in GC non-tumour tissues, HER2-positive tumours and HER2-negative tumours. Tumour HER2 status was determined on formalin-fixed and paraffin-embedded sections by routine immunohistochemistry (IHC) and fluorescent in situ hybridization (FISH) methods, and an IHC score of $3+$ or an IHC score of $2+$ plus FISH with a threshold ratio of more than 2.0 between the HER $2 \mathrm{CN}$ and the chromosome 17 centromere. The average HER2-to-RPPH1 CN ratio of GC non-tumour tissues was set to 1 [standard deviation (SD) 0.2953]. b Tissue rqPCR HER2-to$R P P H 1 \mathrm{CN}$ ratios assessed in GC tumour samples from 48 patients, including 13 with HER2-positive tumours and 35 with HER2negative tumours (bottom). A cut-off value of 2.13 was determined using receiver operating characteristic (ROC) analysis with an area under the curve (AUC) of 0.803 (top). c Comparisons of plasma rqPCR $H E R 2$-to- $R P P H 1 \mathrm{CN}$ ratios ( $Z$ scores) for healthy volunteers and patients with GC with HER2-positive tumours or HER2-negative tumours. $Z$ scores were calculated using the mean and SD of RQ values in a normal plasma panel. Tumour HER2 status was determined as described for a. d Plasma rqPCR HER2-to-RPPH1 $\mathrm{CN}$ ratios ( $Z$ scores) assessed in GC tumour samples from 43 patients, including 13 with HER2-positive tumours and 30 with HER2negative tumours (bottom). A cut-off value of 3.05 was determined using ROC analysis with an AUC of 0.746 (top)

clinicopathological factors. All statistical tests except for paired tests were two sided. Statistical significance was accepted for $P<0.05$.

\section{Results}

Reference gene selection for relative $H E R 2 \mathrm{CN}$ analysis using rqPCR in GC

Our study design for assessing plasma HER2 amplification in patients with GC is shown in Fig. 1. We first optimized our rqPCR assay for HER2 CNs relative to reference gene $\mathrm{CNs}$ in GC. We initially chose three candidate genes, RPPH1 (14q11.2), TAOK1 (17q11.2) and EFTUD2 (17q21.31), on the basis of previous reports [11, 20-22] to avoid false increases or decreases in $H E R 2 \mathrm{CN}$ ratios owing to their normal $\mathrm{CN}$ variations as well as frequent $\mathrm{CN}$ alterations in GC. In microarray-based $\mathrm{CN}$ data obtained from the publically available CCLE database using bioinformatics, the smallest variation was found for RPPHI among these three candidates in $20 \mathrm{GC}$ cell lines $(2.107 \pm 0.4045,2.023 \pm 0.4694$ and $2.207 \pm 0.4617$ for RPPH1, TAOK1 and EFTUD2, respectively; mean $\pm \mathrm{SD}$; Table S3).

In these $20 \mathrm{GC}$ cell lines, the highest correlation between the ratio of $H E R 2$ relative to each reference gene as determined by our rqPCR method and the HER $2 \mathrm{CN}$ data from the CCLE database was for RPPH1 (HER2-toRPPH1 ratio) among these three genes (Fig. S1a). The highest correlation between the ratio of $H E R 2 \mathrm{CN}$ relative to each reference gene and the ratio of HER2 relative to average values of these three reference genes determined in $20 \mathrm{GC}$ cell lines by rqPCR was also for $R P P H 1$ ratios (Fig. S1b). These results suggested that RPPHI would be a useful probe as a reference gene for our HER2 CN analysis in GC. In addition, the stability of the HER2-to$R P P H 1$ ratio was validated using 44 non-tumour DNA samples from patients with GC and 40 normal plasma cfDNA samples (Fig. S1c). The rqPCR HER2-to-RPPHI ratios in serial dilutions starting from $10 \mathrm{ng}$ of a standard sample set prepared by mixing two DNAs without and with HER2 amplification, which were from normal leucocyte DNA and SK-BR-3 cells, respectively, indicated that these results were reproducible up to a 256 -fold dilution (Fig. S1d).

\section{HER2 $\mathrm{CN}$ analysis of GC tissues by rqPCR}

The mean RQ value of the HER2-to-RPPH1 ratios in 44 non-tumour tissues determined by rqPCR was set at 1.00. Then, tumour HER2 $\mathrm{CN}$ was assessed as the $H E R 2$-to-RPPH1 ratio in each GC tissue relative to nontumour tissues. The GC HER2-to-RPPH1 ratios were correlated with IHC HER2 scores $(P=0.000798$, Kruskal-Wallis test; $P=0.0000217$, Jonckheere-Terpstra trend test; Fig. S2a), and were significantly higher in HER2-positive tumours than in HER2-negative tumours $(P=0.0015$, Wilcoxon $t$ test; Fig. 2a). The cut-off value for the tumour HER2-to-RPPH1 ratio to detect $H E R 2$ amplification in GC was determined from ROC analysis with an AUC of 0.803 [95\% confidence interval (CI) 0.653-0.956]. The cut-off value, sensitivity and specificity were $2.13,0.692$ and 0.800 , respectively (Fig. 2b).

The results obtained with the rqPCR HER2-to$R P P H 1$ ratio assay for GC were highly concordant with HER2 status as determined by routine IHC and FISH (concordance of 37/48, 0.771). In addition, the results with the rqPCR HER2-to-RPPH1 ratio assay were highly concordant with the HER2 to chromosome 17 centromere ratio determined by FISH (Fig. S2b). In eight IHC $2+$ tumours, however, two of two FISHpositive tumours (100\%) and two of six FISH-negative tumours $(33.3 \%)$ were tissue rqPCR amplification negative; whereas four of six FISH-negative tumours $(66.7 \%)$ were tissue rqPCR amplification positive (Table S4, Fig. S2b).

Since samples for these two methods were not obtained from the exact same place, these results suggested high intratumoral heterogeneity of $H E R 2 \mathrm{CN}$ and expression in GC tumours, especially in those with borderline HER2 status. 


\section{HER2 amplification detection in circulating cfDNA}

To more objectively evaluate whether the plasma rqPCR HER2-to-RPPH1 ratio (RQ) for an individual patient's sample deviated from the patterns of normal samples, comparative determinations of plasma HER2-to-RPPH1 ratios in patient with $\mathrm{GC}$ were made by setting the mean RQ value of the plasma HER2-to-RPPH1 ratios of 40 healthy volunteers (control samples) to 1.00, after which $Z$ scores were calculated on the basis of the mean and SD of this control sample set [23]. The $Z$ scores of plasma HER2-to-RPPH1 ratios were correlated with IHC HER2 scores using the Kruskal-Wallis and Jonckheere-Terpstra trend tests $(P=0.0443$ and $P=0.00429$, respectively; Fig. S2c), and were significantly higher in patients with HER2-positive tumours than in those with HER2-negative tumours ( $P=0.0116$; Wilcoxon $t$ test; Fig. $2 \mathrm{c})$. The cutoff value for $Z$ scores of this plasma HER2-to- $R P P H 1$ ratio test to detect HER2 amplification in GC was determined using ROC analysis with an AUC of 0.746 (95\% CI 0.560-0.932). The cut-off value, sensitivity and specificity were 3.05, 0.539 and 0.967, respectively (Fig. 2d). In 25 patients in the independent validation cohort, including three patients with HER2-positive tumours, the sensitivity and specificity were 0.667 and 1.000 , respectively, at the cut-off value of 3.05 for $Z$ scores of the plasma HER2-to$R P P H 1$ ratio test (Table $\mathrm{S} 1$ ).

In 39 GC patients, the $Z$ scores for plasma HER2-toRPPH1 ratios were correlated with tumour HER2-toRPPH1 RQ values ( $r=0.424,95 \%$ CI $0.125-0.652$, $P=0.00721)$. A comparison of the $Z$ scores for the plasma HER2-to-RPPH1 ratios before and after surgery in patients from the development and validation cohorts supported the accuracy and reproducibility of this method (Fig. 3). Decreases in the $Z$ scores for plasma HER2-to-RPPH1 ratios after surgery were observed in patients with plasma HER2 amplification before surgery, but not for patients without plasma HER2 amplification in both cohorts, and the decrease observed in HER2-positive patients was statistically significant even though in a small number of patients $(P=0.0122)$.

Possible clinical impact of plasma HER2 testing for GC

The correlations between clinicopathological characteristics of patients with GC and the results of HER2 status determined by routine methods and plasma and tumour rqPCR HER2-to-RPPH1 ratio assays are summarized in Tables 1, 2 and Table S2. Although samples for plasma and/or tumour rqPCR HER2-to-RPPH1 ratio tests were available for only some of the GC patients, the correlations between patient characteristics and tumour HER2 status appeared to be similar among all 52 patients on the basis of
48 patients who were analysed for tissue rqPCR HER2-to$R P P H 1$ ratios and 43 patients who were analysed for plasma rqPCR HER2-to-RPPH1 ratios.

As with routine-method-based tumour HER2 status, the plasma HER2 amplification results tended to be associated with $\mathrm{N}$ stage $(P=0.0637$; Table 2$)$. A similar tendency was found for lymphatic invasion $(P=0.0865$ vs $P=0.0291$ for routine methods vs plasma rqPCR CN assay, respectively). However, unlike routine-method-based tumour HER2 status, the plasma HER2 amplification results were significantly associated with tumour size $(P=0.0236)$. For 13 patients with HER2-positive tumours and whose plasma cfDNA samples were available for rqPCR CN testing, the $Z$ scores of plasma HER2-to- RPPH1 ratios were also associated with tumour size $(P=0.0285$; Table S5). These results suggested that the $Z$ score of the plasma HER2-to-RPPH1 ratio reflected the level of HER2positive cancer cells as well as the degree of HER2 amplification in tumour cells, at least in part.

Discrepancies among HER2 amplification status determined by these three tests were found for some patients (Fig. 4a). By focusing on the discrepancies between routine-method-based tumour HER2 status and plasma rqPCR HER2-to-RPPH1 ratio testing, we made a detailed analysis of subgroups to assess the possible usefulness and limitations of plasma HER2 $\mathrm{CN}$ testing for GC treatment.

For 13 patients who were treated with trastuzumab, 12 were HER 2 positive by routine methods, and seven patients showed plasma HER2 amplification (Table 3). Interestingly, a more effective response to trastuzumab (partial response) was found in all seven patients with plasma HER2 amplification, whereas a less effective response to this treatment (stable disease or progressive disease) was found for four of six patients (three stable disease patients and one progressive disease patient) without plasma HER2 amplification ( $P=0.0210$; Fisher's exact test). We found that rqPCR-based tissue HER2 amplification was not associated with the therapeutic response.

We also assessed the clinical courses of three patients, MK412, MK418 and MK489, whose long-term follow-up along with repeated measurements of plasma HER2 CNs was performed after surgery (Fig. 4b-d). Patient MK489, with an HER2-positive tumour, had a high $Z$ score for the plasma HER2-to-RPPH1 ratio before surgery, and was treated with trastuzumab and docetaxel for recurrence of abdominal lymph node metastasis that was detected by CT at 3 months after surgery (Fig. 4b). This patient had a continuous decrease in the $Z$ score for the plasma HER2-to$R P P H 1$ ratio along with a partial response to treatment for metastatic lymph nodes for more than 2 years. Patient MK412, with an HER2-negative tumour, had a low $Z$ score for the plasma HER2-to-RPPH1 ratio before surgery (Fig. 4c). The $Z$ score for the plasma HER2-to-RPPH1 


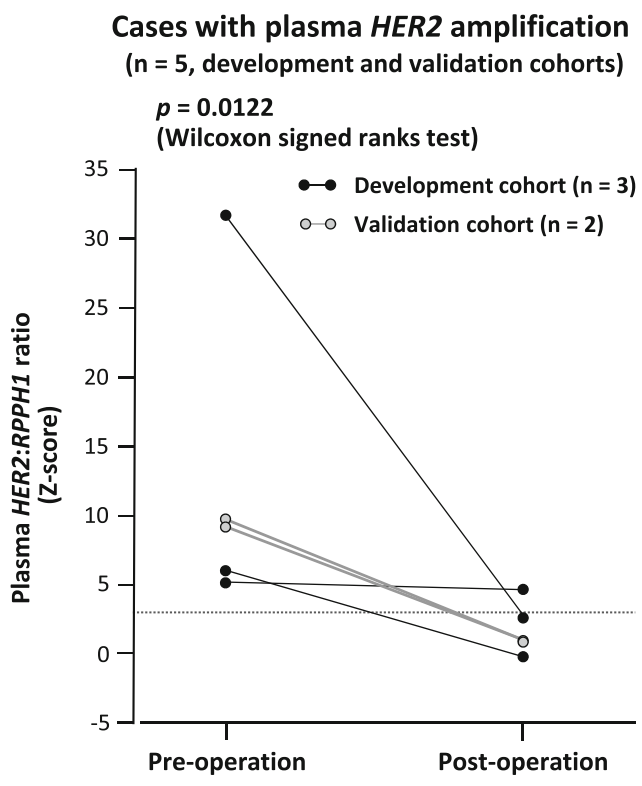

Fig. 3 Comparisons of $Z$ scores for plasma HER2-to- $R P P H 1 \mathrm{CN}$ ratios before and after surgery. Since sets of plasma cfDNA before and after surgery were available only in a limited number of patients, $Z$ scores for plasma $H E R 2$-to-RPPH1 $\mathrm{CN}$ ratios were compared before and after surgery for five patients with GC with plasma HER2 amplification before surgery (left) and 15 patients without plasma

ratio increased gradually and a GC relapse occurred as multiple liver metastases during follow-up. These metastatic tumours showed no response to irinotecan plus cisplatin therapy. Patient MK418, with an HER2-positive tumour, had a low $Z$ score for the plasma HER2-to-RPPH1 ratio before surgery (Fig. $4 \mathrm{~d}$ ). The $Z$ score for the plasma $H E R 2$-to- $R P P H 1$ ratio increased gradually, and a $Z$ score higher than the cut-off value was found on tumour relapse as multiple para-aortic lymph node metastases detected by CT. These metastatic lymph nodes were treated with trastuzumab and capecitabine plus cisplatin, after which a partial response to the treatment was achieved.

\section{Discussion}

Intratumour heterogeneity and incomplete membrane staining patterns in HER2 testing make diagnosing HER2 status more difficult in GC than in BC [24, 25]. HER2 status in GC is determined on the basis of endoscopically biopsied and/or surgically resected specimens, although the heterogeneity of genomic evolution over time has not been considered for trastuzumab treatment. Because solid tumours are known to comprise various subclones, which can show different genomic evolution over time and different responses to treatment [26], HER2 status in GC can change during progression and/or disease recurrence. In $\mathrm{BC}$, the presence of HER2-positive tumour cells in

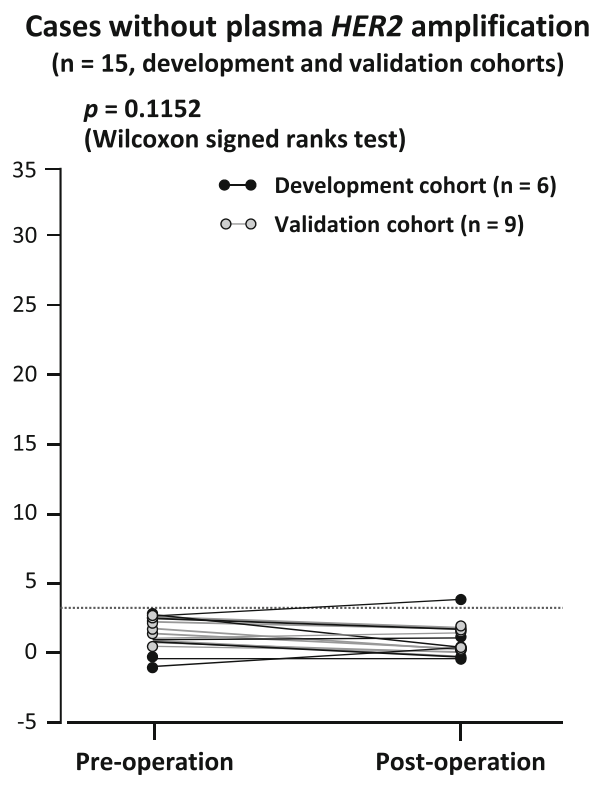

HER2 amplification before surgery (right) from the development and validation cohorts. A similar pattern was observed in both cohorts. Black circles and grey circles represent values for each patient from the development and validation cohorts, respectively, at each time point

recurrent cancer patients with HER2-negative primary tumours has been reported [27]. To overcome these difficulties, in this retrospective pilot study, we investigated the possible utility of plasma cfDNA as an additional source for detecting HER2 amplification and as additive information for predicting the therapeutic effect of trastuzumab treatment in patients with GC.

We have shown that analysing genomic DNA with rqPCR had a high accuracy to determine the HER2 status of tumours and plasma in patients with GC, although our preliminary results need to be validated using larger cohorts of GC patients. First, to circumvent the effects of germline variations and somatic changes on the $\mathrm{CN}$ stability of a reference gene, we assessed candidates for an HER2 rqPCR assay appropriate for GC. Several reference genes for PCR-based plasma HER2 $\mathrm{CN}$ analyses were previously used for $\mathrm{BC}[7,9,11,20]$. However, the unaltered chromosomal regions can be different between GC and BC. In addition, recent studies demonstrated that polysomy 17 could affect HER2 testing and that some BC patients with polysomy 17 should also be considered for therapy with trastuzumab [28-30]. On the basis of previous reports [11, 20-22], we selected three candidates, two on the long arm of chromosome 17 (TKOA1 and EFTUD2) and one on another chromosome (RPPHI). Among them, the CNs of the RPPHI gene appeared to be the stablest in GC cell lines, GC non-tumour tissues and normal plasma samples, suggesting that $R P P H I$ is an appropriate 


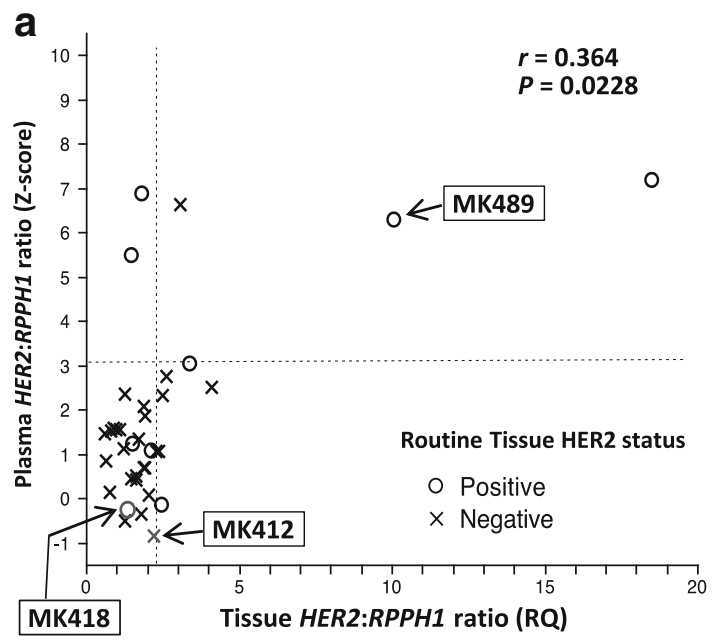

C

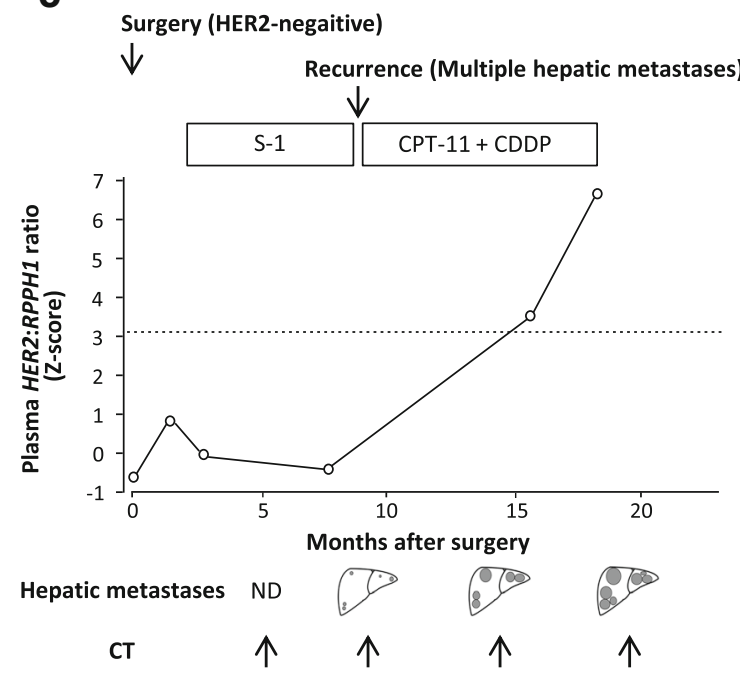

Fig. 4 Detailed analysis of the clinical histories of patients whose plasma HER2 amplification status was monitored repeatedly before and after surgery. a Correlation between tissue rqPCR HER2-to$R P P H 1 \mathrm{CN}$ ratios and $Z$ scores for plasma $H E R 2$-to- $R P P H 1 \mathrm{CN}$ ratios for 39 patients with GC. Circles represent patients with HER2positive tumours and crosses represent patients with HER2-negative tumours. Patients with $Z$ scores for plasma HER2-to-RPPH1 CN ratios ( $y$-axis) of more than 10 and tissue rqPCR HER2-to-RPPH1 CN ratios ( $x$-axis) of more than 20 were excluded from this scatter plot. b Clinical course of patient MK489 with plasma HER2 status monitoring. A 55-year-old female patient underwent surgery for GC of pathological stage T4, N3, M0, stage IIIC and an IHC HER2 score of 3. Although postoperative abdominal lymphatic recurrence (size $16.5 \mathrm{~mm} \times 7.6 \mathrm{~mm}$, shape round) was detected by computed tomography $(C T)$ at 3 months after surgery, a partial response to trastuzumab therapy was achieved at 2 months after starting treatment (size $15.8 \mathrm{~mm} \times 5.0 \mathrm{~mm}$; shape flat). Her plasma $H E R 2 \mathrm{CN}$ was high before surgery, and a decreased level was maintained during treatment with trastuzumab. c Clinical course of patient MK412 with plasma HER2 status monitoring. A 64-year-old male patient b

MK489

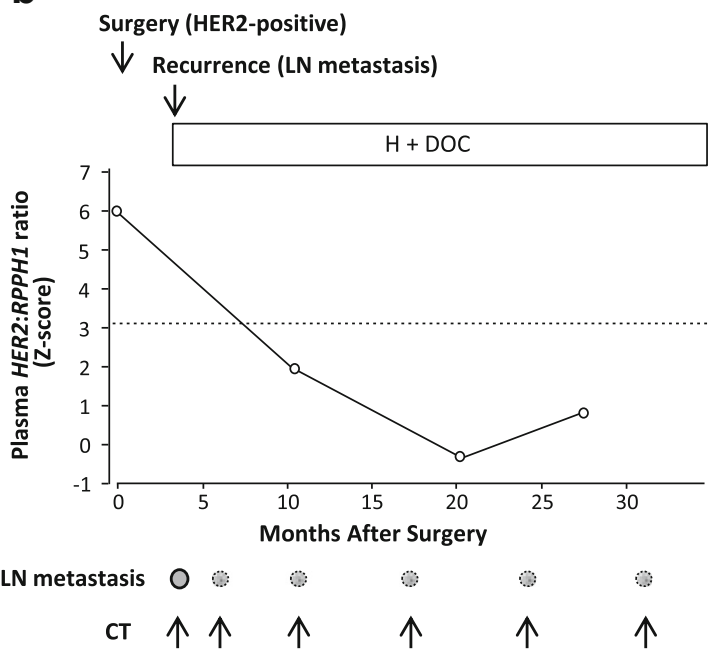

d

MK418

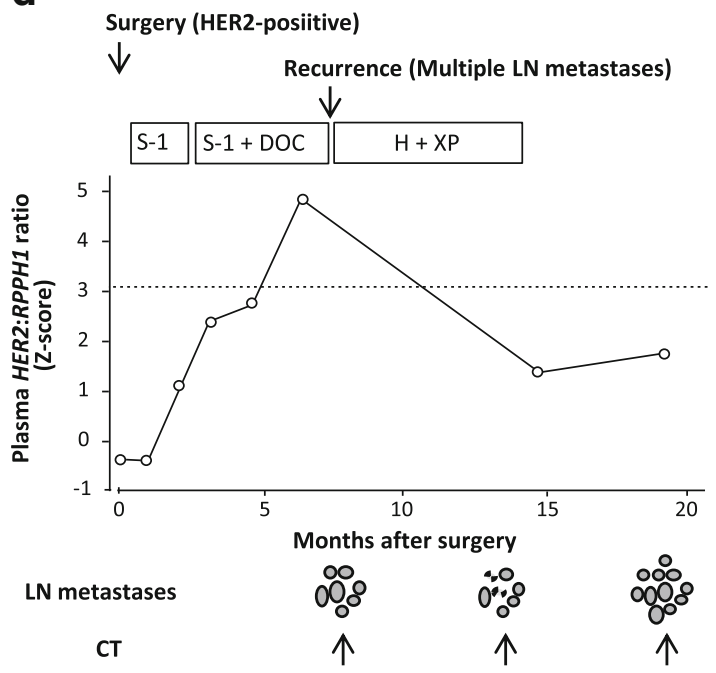

underwent surgery for GC of pathological stage T3N2M0, stage IIIA and an IHC HER 2 score of 2 and negative FISH result. Because his HER2 status was negative, anticancer drugs other than trastuzumab were used for adjuvant chemotherapy. Although his plasma $H E R 2 \mathrm{CN}$ remained at a low level until relapse as multiple hepatic metastases, it increased dramatically after this recurrence. d Clinical course of patient MK412 with plasma HER2 status monitoring. A 52-year-old male patient underwent surgery for GC of pathological stage T4N3M0, stage IIIC and an IHC HER2 score of 3. His plasma HER2 CN was low before surgery, but continuously increased after surgery. After lymphatic recurrence had occurred, trastuzumab therapy was initiated and the plasma $H E R 2 \mathrm{CN}$ declined. Although a partial response to trastuzumab therapy was achieved $(30.4 \%$ reduction) once, renal failure progressed and treatment with this anticancer drug was discontinued. Increases in the sizes and numbers of metastatic lymph nodes were observed along with a slight elevation in plasma HER2 CN. CDDP cisplatin, $C P T-11$ irinotecan, DOC docetaxel, $H$ trastuzumab, $L N$ lymph node, $N D$ none detected, $S-1$ tegafur, gimeracil and oteracil potassium combination, $X P$ capecitabine plus cisplatin 
Table 3 Results of tissue and plasma real-rime quantitative polymerase chain reaction $(r q P C R) H E R 2$-to- $R P P H 1$ copy number $(C N)$ analyses in 13 patients treated with trastuzumab from development cohort

\begin{tabular}{|c|c|c|c|c|c|c|}
\hline \multirow[t]{2}{*}{ Patient $^{\mathrm{a}}$} & \multirow[t]{2}{*}{ Stage $^{b}$} & \multicolumn{2}{|c|}{ Tumour HER2 status $^{c}$} & \multicolumn{2}{|c|}{ rqPCR $H E R 2$-to- $R P P H 1 \mathrm{CN}$ ratio $^{\mathrm{d}}$} & \multirow[t]{2}{*}{ Therapeutic effect of trastuzumab } \\
\hline & & IHC & FISH & Tumour (RQ) & Plasma ( $Z$ score) & \\
\hline MK418 & III & 3 & - & 1.36 & -0.25 & PR \\
\hline MK749 & III & 3 & - & 2.47 & -0.14 & SD \\
\hline MK592 & III & 2 & Negative & NA & 0.28 & PR \\
\hline MK426 & III & 3 & - & 2.13 & 1.08 & SD \\
\hline MK377 & II & 2 & Positive & 1.52 & 1.24 & SD \\
\hline MK614 & III & 3 & - & 39.71 & 2.14 & PD \\
\hline MK618 & III & 3 & - & 3.39 & 3.05 & PR \\
\hline MK564 & IV & 3 & - & NA & 3.07 & PR \\
\hline MK714 & III & 3 & - & 1.48 & 5.49 & PR \\
\hline MK489 & III & 3 & - & 10.07 & 6.29 & PR \\
\hline MK506 & III & 2 & Positive & 1.82 & 6.88 & PR \\
\hline MK694 & III & 3 & - & 18.51 & 7.19 & PR \\
\hline MK624 & III & 3 & - & 22.1 & 33.81 & PR \\
\hline
\end{tabular}

Amplification-positive results are given in bold.

$N A$ not available, $P D$ progressive disease, $P R$ partial response, $R Q$ relative quantitation, $S D$ stable disease

a Trastuzumab therapy for patients with HER2-positive gastric cancer was performed on the basis of the European Medicines Agency criteria, except for patient MK592.

b Disease stage was defined in accordance with the Union for International Cancer Control seventh tumour-lymph node-metastasis (TNM) classification using surgical-pathologic findings.

c Tumour HER2 status was determined by an immumohistochemistry (IHC) score 3+ or an IHC score 2+ with a positive fluorescent in situ hybridization (FISH) result as described in "Materials and Methods".

d Tumour and plasma HER2 amplifications were determined by the rqPCR HER2-to-RPPH1 CN test as described in "Materials and methods".

reference for rqPCR in GC. Unlike BC [20], using a control probe from a chromosome other than chromosome 17 may provide optimal characteristics for HER2 $\mathrm{CN}$ analysis in aneuploid GC cancers for which highly accurate discrimination in $\mathrm{CN}$ is required.

No significant association between the plasma HER2 level and tumour HER2 $\mathrm{CN}$ status determined by FISH has been reported previously [8]. In this study, however, the $Z$ scores of plasma HER2-to-RPPH1 ratios were concordant with the routine-method-based tumour HER2 status, although this degree of concordance was lower than that in BC [20]. High $Z$ scores for plasma HER2-to-RPPH1 ratios were significantly associated with tumour size among all 43 patients and even among 13 patients with positive tissue HER2 status. Among 13 trastuzumab-treated patients, a partial response to therapy was achieved in all seven patients with plasma HER2 amplification, whereas a less effective response (stable disease or progressive disease) was observed in four of six patients without plasma HER2 amplification. No relationship was found between a response to trastuzumab treatment and rqPCR-based tumour HER2 amplification status. Therefore, we hypothesize that plasma rqPCR-based HER2 $\mathrm{CN}$ analyses and routine-method-based and/or rqPCR-based tumour HER2 status have different meanings when used as tools for predicting a therapeutic effect in GC treatment. Because GC tumours, most of which comprise HER2-positive and aggressive tumour cells, could show good responses to molecular targeted therapy for HER2, plasma HER2 CN testing may have clinical utility for predicting GC responsiveness to HER2-targeted therapy.

In this pilot study, we assessed a possible advantage of our plasma rqPCR HER2 CN testing for monitoring HER2 status of GC tumours in a few patients with repeated measurements over time, although the number of patients is insufficient to draw conclusion. In the HER2-positive patient MK418, with a low plasma HER2 CN level before surgery (Fig. 4d), changes in plasma HER2 CN level and clinical course during adjuvant therapy suggested that HER2-amplified GC cells with metastatic potential may not have composed most of the resected tumours, and this potential allowed the emergence of HER2-positive cells. In the HER2-negative patient MK412, with a concordant low plasma HER2 CN level before surgery (Fig. 4c), a continuous increase in plasma $H E R 2 \mathrm{CN}$ level with metastatic recurrence notwithstanding chemotherapy suggested that HER2-amplified GC cells having metastatic potential and resistance to chemotherapeutic reagents may have existed 
as a minor population in primary tumours or that GC cells acquired HER 2 amplification and these phenotypes during tumour progression, and this potential allowed the emergence of HER2-positive cells. It will be interesting to ascertain the reasons for the discordance between HER2 status in biopsied and/or resected tumours and plasma $H E R 2 \mathrm{CN}$ status during the clinical course in a large cohort of patients with GC.

The 'liquid biopsy' method presented in this study using rqPCR as a repeated, non-invasive approach has several advantages for clinical applications [12]. First, a noninvasive blood-based assay is attractive for repeated sampling to evaluate tumour genomic evolution. Second, this method appears to be useful for monitoring therapeutic efficacy and recurrence as a complement to conventional serum tumour markers, such as carcinoembryonic antigen and cancer antigen 19-9, in HER2-positive patients with GC. Another possible application is predicting sensitivity to trastuzumab-based chemotherapy. Our plasma-based HER2 rqPCR testing, rather than tumour HER2 amplification levels [31], may be used to predict the sensitivity to trastuzumab-based combination chemotherapy. In addition, recurrent disease in routine clinical practice is frequently not biopsied when such a biopsy is technically challenging and there are no diagnostic doubts that relapse has occurred. A non-invasive test to screen for acquiring HER2 amplification in these circumstances would be clinically useful.

In conclusion, our preliminary, retrospective study has demonstrated the potential clinical use of circulating cfDNA to detect HER 2 amplification by rqPCR as a therapeutic biomarker in patients with GC. The results obtained using cfDNA did not always correlate with those for a primary tumour, at least partly owing to the spatial and temporal intrinsic heterogeneity of HER2 expression/CN status during GC progression. Further prospective examinations that would focus on the significance of a plasma HER2 test as a non-invasive approach in advanced GC for the purpose of better molecular prediction of therapeutic effects and treatment monitoring during the clinical course of GC will be necessary.

Acknowledgments We thank the Support Center for Advanced Medical Sciences, Institute of Health Biosciences and Institute for Genome Research of the University of Tokushima Graduate School for technical assistance. This work was supported by JSPS KAKENHI grant numbers 23390325 and 25560368 (Issei Imoto), and was partially funded by Chugai Pharmaceutical (Tokyo, Japan) (Eigo Otsuji). The funders had no role in the study design, data collection and interpretation nor in decision to publish or manuscript preparation. All authors contributed to the final manuscript.

Conflict of interest Eigo Otsuji has received research funding from Chugai Pharmaceutical (Tokyo, Japan). All other authors declare that they have no conflict of interest.

\section{References}

1. Siegel R, Ma J, Zou Z, Jemal A. Cancer statistics, 2014. CA Cancer J Clin. 2014. doi:10.3322/caac.21208.

2. Scartozzi M, Galizia E, Verdecchia L, Berardi R, Antognoli S, Chiorrini S, et al. Chemotherapy for advanced gastric cancer: across the years for a standard of care. Expert Opin Pharmacother. 2007;8:797-808.

3. Bang Y-J, Van Cutsem E, Feyereislova A, Chung HC, Shen L, Sawaki A, et al. Trastuzumab in combination with chemotherapy versus chemotherapy alone for treatment of HER2-positive advanced gastric or gastro-oesophageal junction cancer (ToGA): a phase 3, open-label, randomised controlled trial. Lancet. 2010;376:687-97.

4. Gravalos C, Jimeno A. HER2 in gastric cancer: a new prognostic factor and a novel therapeutic target. Ann Oncol. 2008; $19: 1523-9$.

5. Hofmann M, Stoss O, Shi D, Büttner R, van de Vijver M, Kim W, et al. Assessment of a HER2 scoring system for gastric cancer: results from a validation study. Histopathology. 2008;52:797-805.

6. Lee HE, Park KU, Yoo SB, Nam SK, Park DJ, Kim HH, et al. Clinical significance of intratumoral HER2 heterogeneity in gastric cancer. Eur J Cancer. 2013;49:1448-57.

7. Lee HE, Park KU, Yoo SB, Nam SK, Park DJ, Kim HH, et al. Clinical significance of intratumoral HER2 heterogeneity in gastric cancer. Eur J Cancer. 2013;49:1448-57.

8. Rüschoff J, Hanna W, Bilous M, Hofmann M, Osamura RY, Penault-Llorca F, et al. HER2 testing in gastric cancer: a practical approach. Mod Pathol. 2012;25:637-50.

9. Bechmann T, Andersen RF, Pallisgaard N, Madsen JS, Maae E, Jakobsen EH, et al. Plasma HER2 amplification in cell-free DNA during neoadjuvant chemotherapy in breast cancer. J Cancer Res Clin Oncol. 2013;139:995-1003.

10. Murtaza M, Dawson SJ, Tsui DW, Gale D, Forshew T, Piskorz $\mathrm{AM}$, et al. Non-invasive analysis of acquired resistance to cancer therapy by sequencing of plasma DNA. Nature. 2013;497:108-12.

11. Page K, Hava N, Ward B, Brown J, Guttery DS, Ruangpratheep $\mathrm{C}$, et al. Detection of HER2 amplification in circulating free DNA in patients with breast cancer. Br J Cancer. 2011;104:1342-8.

12. Tsujiura M, Ichikawa D, Konishi H, Komatsu S, Shinozaki AD, Otsuji E. Liquid biopsy of gastric cancer patients: circulating tumor cells and cell-free nucleic acids. World J Gastroenterol. 2014;20:3265-86.

13. Sorensen BS, Mortensen LS, Andersen J, Nexo E. Circulating HER2 DNA after trastuzumab treatment predicts survival and response in breast cancer. Anticancer Res. 2010;30:2463-8.

14. Ichikawa D, Koike H, Ikoma H, Ikoma D, Tani N, Otsuji E, et al. Detection of aberrant methylation as a tumor marker in serum of patients with gastric cancer. Anticancer Res. 2004;24:2477-81.

15. Park KU, Lee HE, Park DJ, Jung EJ, Song J, Kim HH, et al. MYC quantitation in cell-free plasma DNA by real-time PCR for gastric cancer diagnosis. Clin Chem Lab Med. 2009;47:530-6.

16. Eisenhauer EA, Therasse P, Bogaerts J, Schwartz LH, Sargent D, Ford R, et al. New response evaluation criteria in solid tumours: revised RECIST guideline (version 1.1). Eur J Cancer. 2009;45:228-47.

17. Sobin LH, Wittekind $\mathrm{CH}$, Gospodarowicz M, editors. TNM classification of malignant tumors. 7th ed. New York: Wiley; 2009. p. 73-7.

18. Tomita H, Ichikawa D, Ikoma D, Sai S, Tani N, Ikoma H, et al. Quantification of circulating plasma DNA fragments as tumor markers in patients with esophageal cancer. Anticancer Res. 2007;27:2737-42. 
19. Akobeng AK. Understanding diagnostic tests 3: receiver operating characteristic curves. Acta Paediatr. 2007;96:644-7.

20. Gevensleben H, Garcia-Murillas I, Graeser MK, Schiavon G, Osin P, Parton M, et al. Noninvasive detection of HER2 amplification with plasma DNA digital PCR. Clin Cancer Res. 2013;19:3276-84.

21. Jacquemier J, Spyratos F, Esterni B, Mozziconacci MJ, Antoine $\mathrm{M}$, Arnould L, et al. SISH/CISH or qPCR as alternative techniques to FISH for determination of HER2 amplification status on breast tumors core needle biopsies: a multicenter experience based on 840 cases. BMC Cancer. 2013;13:351.

22. Takada H, Imoto I, Tsuda H, Sonoda I, Ichikura T, Mochizuki H, et al. Screening of DNA copy-number aberrations in gastric cancer cell lines by array-based comparative genomic hybridization. Cancer Sci. 2005;96:100-10.

23. Leary RJ, Sausen M, Kinde I, Papadopoulos N, Carpten JD, Craig $\mathrm{D}$, et al. Detection of chromosomal alterations in the circulation of cancer patients with whole-genome sequencing. Sci Transl Med. 2012;162:1-12.

24. Cho EY, Park K, Do I, Cho J, Kim J, Lee J, et al. Heterogeneity of ERBB2 in gastric carcinomas: a study of tissue microarray and matched primary and metastatic carcinomas. Mod Pathol. 2013;26:677-84.

25. Okines AF, Cunningham D. Trastuzumab in gastric cancer. Eur J Cancer. 2010;46:1949-59.
26. Onsum MD, Geretti E, Paragas V, Kudla AJ, Moulis SP, Luus L, et al. Single-cell quantitative HER2 measurement identifies heterogeneity and distinct subgroups within traditionally defined HER2-positive patients. Am J Pathol. 2013;183:1446-60.

27. Hayashi N, Nakamura S, Tokuda Y, Shimoda Y, Yagata H, Yoshida A, et al. Prognostic value of HER2-positive circulating tumor cells in patients with metastatic breast cancer. Int J Clin Oncol. 2012;17:96-104.

28. Hanna WM, Rüschoff J, Bilous M, Coudry RA, Dowsett M, Osamura RY, et al. HER2 in situ hybridization in breast cancer: clinical implications of polysomy 17 and genetic heterogeneity. Mod Pathol. 2014;27:4-18.

29. Krishnamurti U, Hammers JL, Atem FD, Storto PD, Silverman JF. Poor prognostic significance of unamplified chromosome 17 polysomy in invasive breast carcinoma. Mod Pathol. 2009;22:1044-8.

30. Vanden Bempt I, van Loo P, Drijkoningen M, Neven P, Smeets A, Christiaens MR, et al. Polysomy 17 in breast cancer: clinicopathologic significance and impact on HER-2 testing. J Clin Oncol. 2008;26:4869-74.

31. Gomez-Martin C, Plaza JC, Pazo-Cid R, Salud A, Pons F, Fonseca $\mathrm{P}$, et al. Level of HER2 gene amplification predicts response and overall survival in HER2-positive advanced gastric cancer treated with trastuzumab. J Clin Oncol. 2013;31:4445-52. 